\title{
Effects of Grazing and Sagebrush Control on Potential Erosion
}

\author{
C.W. JOHNSON, G.A. SCHUMAKER, AND J.P. SMITH
}

\begin{abstract}
Canopy and ground cover data from 1972 through 1978 were used in the Universal Soil Loss Equation to compute potential soil loss on grazed and ungrazed areas subjected to brush treatment and no treatment at nine sites on the Reynolds Creek Experimental Watershed in Southwest Idaho. Results showed the computed soil loss differences between grazed and ungrazed areas were not significant $(P>.05)$ on five sites, were significant $(P<.05)$ on two sites, and were highly significant $(P<.01)$ on two sites. Soil loss differences between sagebrush and eradication treatments and untreated areas were not significant $(P>.05)$ on four sprayed areas, but were highly significant $(P<.01)$ on two areas where brush was cut and removed. Sediment delivery ratios, based on measured watershed sediment yield and computed soil loss, ranged from 0.15 to 0.47 .
\end{abstract}

Because of past overgrazing and fires, soil erosion is a major concern on about 39 million ha of sagebrushdominated rangeland in the western United States (Tisdale et al. 1969). Cattle grazing is a major use in most of this area, but sheep, wild horses, and other animals graze it as wellall compete for the available forage. Grazing management, brush cradication, and range seeding are the most common practices used to increase grazing capacity.

The Universal Soil Loss Equation, USLE, has been adapted for estimating soil loss from rangelands and other undisturbed areas by Wischmeier $(1975,1976)$, Wischmeier and Smith (1978), Jurinak et al. (1977), and the U.S. Department of Agriculture (1975). However, soil loss estimates on rangelands are less reliable than on croplands due to lack of research (Wischmeier 1976). McCool et al. (1976) also developed a method for using the USLE in the Pacific Northwest. These equations and methods were used in this study to compute potential soil loss from grazed and ungrazed areas and to determine the effects of sagebrush eradication treatments on the USLE cover and management factor and resulting soil loss. Estimated onsite soil losses were compared with watershed sediment yields.

\section{Study Areas and Methods}

Nine study sites, which represented a wide range of vegetation litter, rock, and brush canopy cover and precipitation, soils, and topography on the Reynolds Creek Experimental Watershed, 80 km southwest of Boise, Ida., were selected for this study (see Table 1). Areas at each site were fenced before the 1972 grazing season to exclude cattle and to measure various types of cover for application of the USLE on grazed, ungrazed, and treated areas. Forage utilization was estimated on grazed areas during the study period. Big sagebrush (Artemisia tridentata wyomingensis and A.T.

\footnotetext{
Authors are hydraulic engineer, soil scientist, and hydrologic technician, respectively; Northwest Watershed Research Center, U.S. Dep. Agr. Sci. Educ. Admin. Agr. Res., Suite 116, 1175 South Orchard, Boise, Idaho 83705.

This research is a contribution from the Northwest Watershed Research Center, Sci. and Educ. Admin. Agr. Res., U.S. Dep. Agr., and Bureau of Land Management. U.S. Dept. of Interior in cooperation with the Agricultural Experiment Station, University of Idaho. Moscow.

Manuscript received April 23, 1979.
}

vaseyana) were killed by spraying with 2,4-D[2,4-dichlorophenoxy) acetic acid] at Nancy, Whiskey, and Reynolds Mountain east sites, and with 2,4,5-T[2,4,5-trichlorophenoxy) acetic acid] at the Upper Sheep north facing site. Big sagebrush was cut and removed on three of these sites. The sites where sagebrush was killed were not seeded. The Nettleton site was very severely grazed to determine the effects of $80-100 \%$ forage utilization on vegetal, litter, and brush canopy cover and soil loss.

A spoked-wheel adaptation of the step-point sampling method, described by Evans and Love (1957), was used to determine bare ground, large and small rock, litter, and vegetal ground cover and canopy by species at $500-700$ transect points on grazed, ungrazed, and brush eradication areas. Transects were sampled each year, 1972-78, when vegetal cover was near maximum and plants were most easily identified. Also, additional transects were made near the end of the grazing season in 1974, 1975, and 1978.

The Universal Soil Loss Equation in metric units is

$$
A=2.242 R K L S C P
$$

where $A$ is soil loss in metric tonnes/ha/year, $R$ is the rainfallrunoff factor, $K$ is the soil erodibility factor, $L S$ is the slope length-gradient factor, $C$ is the cover and management factor, and $P$ is the erosion control-practices factor.

Values of the $R$-factor were determined by the equation in metric units.

$$
R=0.0219 P_{\mathrm{f}}^{2.2}+0.0591 P_{(\mathrm{D}-\mathrm{M})}
$$

where, $P_{f}$ is the 2-yr 6-hr precipitation in mm at the site (Miller et al. 1973) and $P_{(\mathrm{D}-\mathrm{M})}$ is the December through March precipitation in $\mathrm{mm}$ at the site. Values of the $K$ factor were determined for each site by using the Reynolds Creek Watershed soil map (Stephenson 1977) and the soil erodibility values assigned by the U.S. Department of Agriculture (1974). Values for the slope length-gradient factor, $L S$, were determined by using site information and the slope length-steepness relationship developed by $\mathrm{McCool}$ et al. (1976). Values for the cover and management factor, $C$, were determined near the beginning of the grazing season from transect data using procedures developed by Wischmeier (1975). The erosion-control practices, $P$, was set equal to one in the computations, because there were not applicable practices in this study. Analysis of variance was used to test the significance between differences in computed soil loss and vegetal, litter, and canopy cover on grazed and ungrazed areas and on brush control treatments.

\section{Results and Discussion}

Average precipitation, cover, and USLE factor values for the grazed areas at the study sites are listed in Table 2 . The wide range in $R$-values mainly reflects the increase in yearly precipitation from $250 \mathrm{~mm}$ at the Flats, where elevation is $1,190 \mathrm{~m}$, to $1,070 \mathrm{~mm}$ at Reynolds Mountain, where elevation is $2,090 \mathrm{~m}$. The lower $K$-values at Nancy, Lower Sheep, Upper Sheep south facing, and Reynolds Mountain west sites are due mainly to the very rocky soils. The $C$-values, determined at each site from cover transect data, were much 
Table 1. Description of study sites on Reynolds Creek Experimental Watershed.

\begin{tabular}{|c|c|c|c|c|}
\hline Site name & $\begin{array}{l}\text { Elevation } \\
\quad(\mathrm{m})\end{array}$ & $\begin{array}{l}\text { Grazing } \\
\text { intensity }\end{array}$ & $\begin{array}{c}\text { Soil } \\
\text { (Series) }\end{array}$ & Vegetation association $^{2}$ \\
\hline \multicolumn{5}{|l|}{ Big sagebrush: } \\
\hline Flats & 1190 & Heavy & Nannyton loam & $\begin{array}{l}\text { Shadscale (Artiplex confertifolia), big sage- } \\
\text { brush (Artemisia tridentata wyomingensis) }\end{array}$ \\
\hline Nancy & 1410 & Heavy & Ruclick and Babbington stony gravelly loam & $\begin{array}{l}\text { Big sagebrush (A.T. wyomingensis), bearded } \\
\text { bluebunch wheatgrass (Agropyron spicatum) }\end{array}$ \\
\hline Whiskey & 1700 & Heavy & Takeuchi rocky coarse sandy loam & $\begin{array}{l}\text { Big sagebrush (A.T. vaseyana), bitterbrush } \\
\text { (Purshia tridentata) }\end{array}$ \\
\hline $\begin{array}{l}\text { Upper Sheep, } \\
\text { north facing }\end{array}$ & 1880 & Heavy & Harmehl and Demast stony loam & $\begin{array}{l}\text { Big sagebrush ( } A . T \text {. vaseyana), snowberry } \\
\text { (Symphoricarpos oreophilus) }\end{array}$ \\
\hline $\begin{array}{l}\text { Reynolds Mtn., } \\
\text { east }\end{array}$ & 2090 & Moderate & Bullrey gravelly loam & Big sagebrush (A.T. vaseyana) \\
\hline Nettleton & 1500 & Severe & Reywat-Bakeoven rocky very stony loam & $\begin{array}{l}\text { Big sagebrush (A.T. wyomingensis), bearded } \\
\text { bluebunch wheatgrass (Agropyron spicatum) }\end{array}$ \\
\hline \multicolumn{5}{|c|}{ Low-growing sagebrush } \\
\hline Lower Sheep & 1620 & Heavy & Searla gravelly loam & $\begin{array}{l}\text { Low sagebrush (Artemisia arbuscula), sand- } \\
\text { berg bluegrass (Poa secunda) }\end{array}$ \\
\hline $\begin{array}{l}\text { Upper Sheep, } \\
\text { south facing }\end{array}$ & 1880 & Heavy & Gabica cobbly gravelly loam & $\begin{array}{l}\text { Low sagebrush (Artemisia arbuscula), sand- } \\
\text { berg bluegrass (Poa secunda) }\end{array}$ \\
\hline $\begin{array}{l}\text { Reynolds Mt., } \\
\text { west }\end{array}$ & 2090 & Moderate & Bullrey gravelly loam & $\begin{array}{l}\text { Big sagebrush (A.T. wyomingensis), }{ }^{3} \text { idaho } \\
\text { fescue (Festuca idahoensis) }\end{array}$ \\
\hline
\end{tabular}

IGrazing intensity scale; Moderate $=41-60 \%$ utilization; heavy $=61-80 \%$ utilization, and severe $=81-100 \%$ utilization of key forage species.

${ }^{2}$ Generally, Big sagebrush Artemisia tridentata) was about $0.6 \mathrm{~m}$ high, and low sagebrush (Artemisia arbuscula) was about $0.25 \mathrm{~m}$ high.

${ }^{3}$ Big sagebrush at this site was about $0.25 \mathrm{~m}$ high and looked much like low sagebrush.

greater at the Flats and Nancy sites with low precipitation. Total ground cover at the end of the grazing season was not significantly different from cover measurements made earlier at optimum growth, therefore, early grazing season transect data were used in this study. The wide range in slope length-gradient factor, $L S$, values explains most of the differences in computed soil loss between sites and shows why erosion is often so evident on long steep slopes.

Since the only factor in the USLE that can be affected by grazing is the cover and management factor, $C$, soil loss was computed for grazed and ungrazed areas at each site by the USLE to determine the influence of grazing on predicted soil loss. Table 3 compares the computed yearly soil losses, which were determined from yearly cover transects and longterm $R, K$, and $L S$ values shown in Table 2 . Analysis of variance on computed soil losses showed no significant differences $(P>.05)$ between heavily or moderately grazed and ungrazed areas at five sites for the 7-yr study period. Most sites, however, showed a considerable year-to-year difference in computed soil loss, because of complex interactions of vegetal, litter, and brush canopy cover, climate, and forage utilization. Computed soil losses on Upper Sheep sites were significantly different $(P<.05)$, probably because the site is highly productive (Schumaker and Hanson 1977)

Table 2. Average precipitation, cover, and USLE factor values for grazed areas at study sites, 1972-78 cover transects.

\begin{tabular}{|c|c|c|c|c|c|c|c|c|c|c|c|}
\hline \multirow{4}{*}{ Site } & \multirow{4}{*}{$\begin{array}{c}\text { Precipi- } \\
\text { tation' } \\
(\mathrm{mm})\end{array}$} & \multirow{2}{*}{\multicolumn{4}{|c|}{ Cover $\%$}} & \multicolumn{6}{|c|}{ USLE Factor } \\
\hline & & & & & & \multirow{3}{*}{$\begin{array}{c}\text { Rainfall } \\
\text {-Runoff } \\
\text { (R) }\end{array}$} & \multirow{3}{*}{$\begin{array}{l}\text { Soil } \\
\text { erodi- } \\
\text { bility } \\
(\mathbf{K})\end{array}$} & \multirow{3}{*}{$\begin{array}{l}\text { Slope } \\
\text { length- } \\
\text { gradient } \\
\text { (LS) }\end{array}$} & \multirow{3}{*}{$\begin{array}{l}\text { Cover } \\
\text { (C) }\end{array}$} & \multirow{2}{*}{\multicolumn{2}{|c|}{ Soil loss ${ }^{1,2}$}} \\
\hline & & & & & Brush & & & & & & \\
\hline & & Vegetal & Litter & Rock & canopy & & & & & $\mathrm{t} / \mathrm{a} / \mathrm{yr}$ & $\mathrm{t} / \mathrm{ha} / \mathrm{yr}$ \\
\hline \multicolumn{12}{|l|}{ Big sagebrush } \\
\hline Flats & 250 & 10.7 & 20.8 & 11.4 & 9.2 & 17.9 & 0.28 & 0.7 & 0.094 & 0.33 & 0.74 \\
\hline Nancy & 280 & 23.1 & 22.9 & 17.4 & 11.2 & 20.9 & 0.20 & 1.4 & 0.054 & 0.32 & 0.71 \\
\hline Whiskey & 510 & 19.7 & 45.9 & 5.1 & 32.8 & 29.9 & 0.15 & 2.8 & 0.027 & 0.34 & 0.76 \\
\hline Upper Sheep, north facing & 410 & 21.8 & 61.6 & 0.8 & 31.7 & 28.0 & 0.28 & 9.3 & 0.018 & 1.31 & 2.94 \\
\hline Reynolds Mtn., east facing & 1070 & 21.4 & 53.9 & 3.7 & 36.7 & 56.3 & 0.28 & 1.4 & 0.020 & 0.44 & 0.99 \\
\hline Nettleton & 500 & 19.9 & 36.4 & 13.1 & 8.0 & 37.0 & 0.28 & 4.6 & 0.029 & 1.38 & 3.10 \\
\hline \multicolumn{12}{|l|}{ Low-growing sagebrush } \\
\hline Lower Sheep & 350 & 26.1 & 18.1 & 31.5 & 22.3 & 25.4 & 0.15 & 4.3 & 0.024 & 0.39 & 0.88 \\
\hline Upper Sheep, south facing & 410 & 10.7 & 10.7 & 36.1 & 22.3 & 28.0 & 0.15 & 7.6 & 0.049 & 1.56 & 3.50 \\
\hline Reynolds Mtn., west ${ }^{4}$ & 1070 & 19.8 & 19.0 & 42.5 & 10.2 & 56.3 & 0.17 & 1.7 & 0.020 & 0.33 & 0.73 \\
\hline
\end{tabular}

11963-78 Precipitation

${ }^{2}$ Tons per acre pre year

${ }^{3}$ Metric tonnes per hectare per year

${ }^{4}$ Sagebrush at this site is about $0.25 \mathrm{~m}$ high and looks much like low sagebrush (Artemisia arbuscula), but it has been identified as a low-growing big sagebrush (Artemisia tridentata wyomingensis). 


\begin{tabular}{|c|c|c|c|c|c|c|c|c|}
\hline \multirow[b]{2}{*}{ Site and treatment } & \multicolumn{7}{|c|}{ Year } & \multirow[b]{2}{*}{ Mean } \\
\hline & 1972 & 1973 & 1974 & 1975 & 1976 & 1977 & 1978 & \\
\hline \multicolumn{9}{|l|}{ Big sagebrush } \\
\hline $\begin{array}{l}\text { Flats } \\
\text { Ungrazed } \\
\text { Grazed }\end{array}$ & $\begin{array}{l}0.54 \\
0.56\end{array}$ & $\begin{array}{l}0.74 \\
0.74\end{array}$ & $\begin{array}{l}0.29 \\
0.40\end{array}$ & $\begin{array}{l}1.05 \\
0.90\end{array}$ & $\begin{array}{l}1.66 \\
1.10\end{array}$ & $\begin{array}{l}0.38 \\
0.69\end{array}$ & $\begin{array}{l}0.56 \\
0.83\end{array}$ & $\begin{array}{l}0.75 \\
0.75 \mathrm{NS}\end{array}$ \\
\hline $\begin{array}{l}\text { Nancy } \\
\text { Ungrazed } \\
\text { Grazed }\end{array}$ & $\begin{array}{l}0.52 \\
0.43\end{array}$ & $\begin{array}{l}0.63 \\
0.94\end{array}$ & $\begin{array}{l}0.49 \\
0.67\end{array}$ & $\begin{array}{l}0.58 \\
0.74\end{array}$ & $\begin{array}{l}0.72 \\
0.78\end{array}$ & $\begin{array}{l}0.49 \\
0.58\end{array}$ & $\begin{array}{l}0.78 \\
0.83\end{array}$ & $\begin{array}{l}0.60 \\
0.71 \mathrm{NS}\end{array}$ \\
\hline $\begin{array}{l}\text { Whiskey } \\
\text { Ungrazed } \\
\text { Grazed }\end{array}$ & $\begin{array}{l}0.76 \\
0.69\end{array}$ & $\begin{array}{l}0.74 \\
0.63\end{array}$ & $\begin{array}{l}0.69 \\
0.85\end{array}$ & $\begin{array}{l}0.63 \\
0.67\end{array}$ & $\begin{array}{l}0.85 \\
0.69\end{array}$ & $\begin{array}{l}1.01 \\
0.76\end{array}$ & $\begin{array}{l}0.67 \\
0.96\end{array}$ & $\begin{array}{l}0.76 \\
0.75 \mathrm{NS}\end{array}$ \\
\hline $\begin{array}{l}\text { Upper Sheep, N.F } \\
\text { Ungrazed } \\
\text { Grazed }\end{array}$ & $\begin{array}{l}0.65 \\
1.48\end{array}$ & $\begin{array}{l}1.64 \\
2.28\end{array}$ & $\begin{array}{l}1.14 \\
3.76\end{array}$ & $\begin{array}{l}0.81 \\
2.28\end{array}$ & $\begin{array}{l}1.30 \\
5.46\end{array}$ & $\begin{array}{l}1.95 \\
2.13\end{array}$ & $\begin{array}{l}0.65 \\
3.27\end{array}$ & $\begin{array}{l}1.16 \\
2.95^{*}\end{array}$ \\
\hline $\begin{array}{l}\text { Reynolds Mtn., E } \\
\text { Ungrazed } \\
\text { Grazed }\end{array}$ & $\begin{array}{l}0.69 \\
0.85\end{array}$ & $\begin{array}{l}0.74 \\
0.90\end{array}$ & $\begin{array}{l}1.28 \\
1.19\end{array}$ & $\begin{array}{l}0.74 \\
0.94\end{array}$ & $\begin{array}{l}0.49 \\
1.48\end{array}$ & $\begin{array}{l}0.49 \\
0.74\end{array}$ & $\begin{array}{l}0.49 \\
0.85\end{array}$ & $\begin{array}{l}0.70 \\
0.99 \mathrm{NS}\end{array}$ \\
\hline $\begin{array}{l}\text { Nettleton } \\
\text { Ungrazed } \\
\text { Grazed }\end{array}$ & $\begin{array}{l}1.70 \\
2.46\end{array}$ & $\begin{array}{l}1.81 \\
2.35\end{array}$ & $\begin{array}{l}1.19 \\
2.24\end{array}$ & $\begin{array}{l}0.65 \\
3.20\end{array}$ & $\begin{array}{l}2.24 \\
5.47\end{array}$ & $\begin{array}{l}1.28 \\
2.15\end{array}$ & $\begin{array}{l}0.85 \\
3.74\end{array}$ & $\begin{array}{l}1.39 \\
3.09^{* *}\end{array}$ \\
\hline Low-growing sagebr & & & & & & & & \\
\hline $\begin{array}{l}\text { Lower Sheep } \\
\text { Ungrazed } \\
\text { Grazed }\end{array}$ & $\begin{array}{l}0.76 \\
0.76\end{array}$ & & $\begin{array}{l}0.76 \\
0.85\end{array}$ & $\begin{array}{l}0.99 \\
1.14\end{array}$ & $\begin{array}{l}1.17 \\
1.08\end{array}$ & $\begin{array}{l}0.56 \\
0.76\end{array}$ & $\begin{array}{l}0.76 \\
0.76\end{array}$ & $\begin{array}{l}0.83 \\
0.89 \mathrm{NS}\end{array}$ \\
\hline $\begin{array}{l}\text { Upper Sheep, S.F. } \\
\text { Ungrazed } \\
\text { Grazed }\end{array}$ & $\begin{array}{l}2.29 \\
3.00\end{array}$ & & $\begin{array}{l}3.72 \\
3.58\end{array}$ & $\begin{array}{l}2.78 \\
3.79\end{array}$ & $\begin{array}{l}3.23 \\
4.21\end{array}$ & $\begin{array}{l}2.58 \\
3.58\end{array}$ & $\begin{array}{l}2.35 \\
2.93\end{array}$ & $\begin{array}{l}2.83 \\
3.52^{*}\end{array}$ \\
\hline $\begin{array}{l}\text { Reynolds Mtn., W } \\
\text { Ungrazed } \\
\text { Grazed }\end{array}$ & $\begin{array}{l}0.52 \\
0.81\end{array}$ & & $\begin{array}{l}0.63 \\
0.74\end{array}$ & $\begin{array}{l}0.54 \\
0.76\end{array}$ & $\begin{array}{l}0.54 \\
0.63\end{array}$ & $\begin{array}{l}0.69 \\
0.65\end{array}$ & $\begin{array}{l}0.59 \\
0.76\end{array}$ & $0.73^{* *}$ \\
\hline
\end{tabular}

NS Difference not significant $(P>.05)$

* Difference significant $(P<.05)$

** Difference significant $(P<.01)$

and thus is subjected to greater trampling and cover reduction than the other sites except Nettleton. Computed soil losses for Upper Sheep sites with steep slopes were much higher than for most other sites; the steep slopes magnified the soil loss differences between grazed and ungrazed areas. The computed soil loss difference at the moderately grazed Reynolds Mountain west site was highly significant $(P<.01)$, although no reasons for this disparate condition were found. The computed soil loss difference between the severely grazed and ungrazed areas at the Nettleton site was highly significant $(P<.01)$ - the computed soil loss increased from 1.38 metric tonnes/ha/ $\mathrm{yr}$ in the ungrazed area to 3.10 metric tonnes/ha/yr in the grazed area.

Percentage vegetal cover and bare ground data from grazed and ungrazed areas (Table 4) showed that on combined big sagebrush sites, litter cover was about $40 \%$ of total ground cover on grazed and $43 \%$ on ungrazed areas; that vegetal cover was about $19 \%$ on grazed and $23 \%$ on ungrazed areas; that canopy cover was about $22 \%$ on grazed and $23 \%$ on ungrazed areas; and that about $32 \%$ of the grazed and $27 \%$ of the ungrazed areas were bare ground. The only sites where the difference in bare ground was significant $(P<.05)$ between grazed and ungrazed areas were Nettleton, severely grazed, and Upper Sheep south facing sites. Sites with low-growing sagebrush (Table 4) had similar percentage bare ground and less vegetative cover than big sagebrush sites; however, the rocky soils on low-growing sagebrush sites are more erosion resistant. Generally, the percentage bare ground was slightly greater on grazed than ungrazed areas. The 7-yr study period was not sufficient to establish a reliable soil loss trend; nevertheless, a consistent slight reduction in cover due to grazing was evident.

The computed yearly soil loss from 1972-75 in ungrazed areas at four big sagebrush sites with brush control treatments is summarized in Table 5. The combined results showed that soil loss from sprayed areas was not significantly different $(P<.05)$ from untreated areas, but that brush-cut areas had 1.07 metric/tonnes/ha greater [highly significant $(P<.01)]$ soil loss than untreated areas. The soil loss difference between untreated and sagebrush-cut areas was highly significant $(P<.01)$ at two sites. Yearly valucs ranged widely in response to climatic, site, and treatment differences. These results showed that eradication of sagebrush by spraying did not increase the potential for soil loss significantly $(P>.05)$; however, where sagebrush was cut and removed, the potential soil loss increase was highly significant $(P<.01)$.

Six of the sites used in this study are located within four watersheds where sediment yield has been measured (Johnson and Hanson 1976). The USLE was used to compute average soil loss on the watersheds for comparison with sediment yield. In Table 6, soil loss on study sites and 
Table 4. Percentage litter, vegetal, and brush canopy cover and bare ground at Reynolds Creek sites, 1972-78 average values.

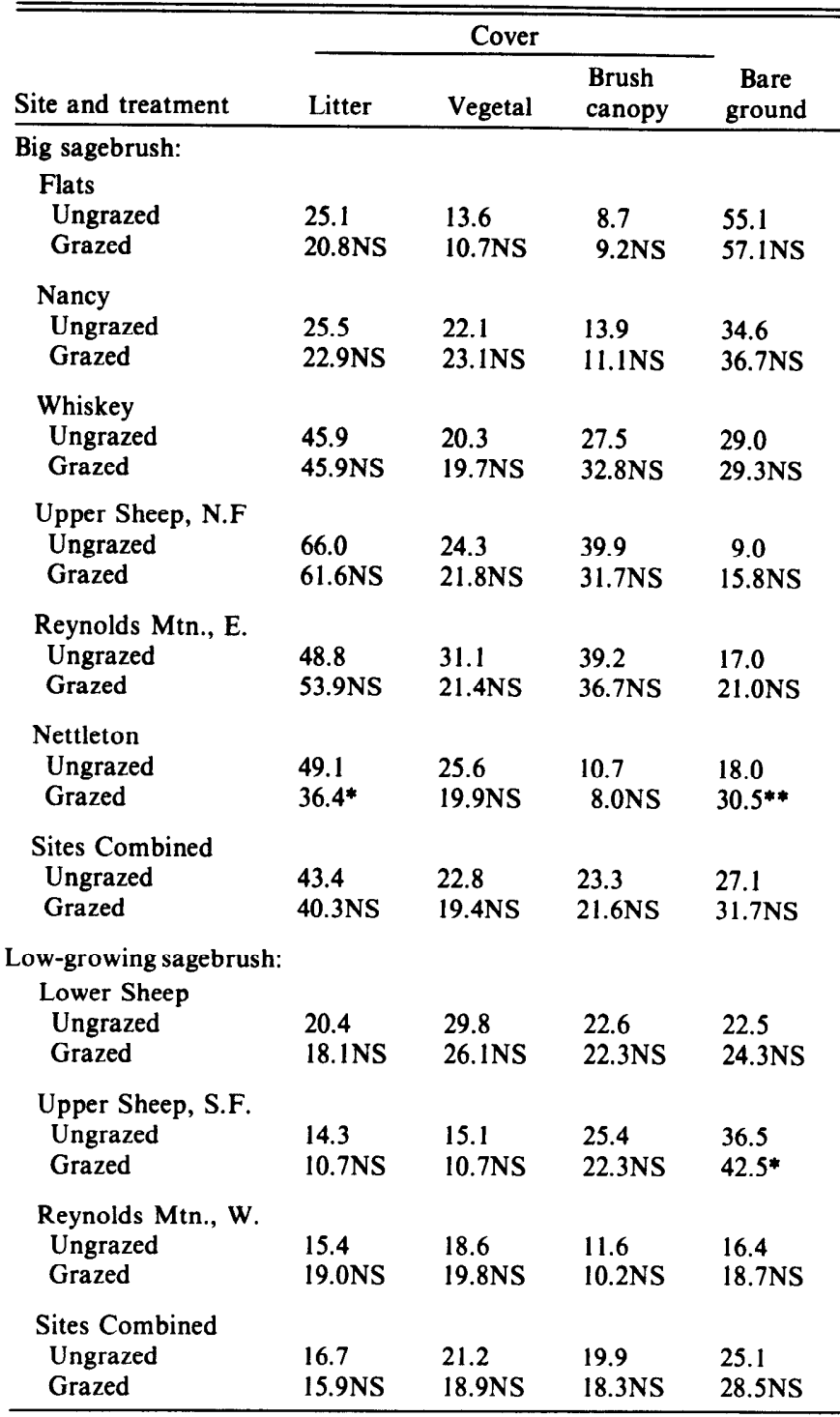

NS Differences in cover on grazed and ungrazed areas not significant $(P>05)$

* Differences significant $(P<.05)$

** Differences highly significant $(P<.01)$

watersheds computed by the USLE is compared with measured watershed sediment yield. Sediment yields, which were the average of 5 to 10 years of record at watershed stations, averaged only about $25 \%$ of computed onsite soil loss.

Additional research is needed in applying the USLE to rangelands; however, the procedure is useful in comparing the effects of various treatments and management practices on potential onsite erosion. As additional data become available, the USLE should be used with more confidence in rangeland erosion studies.

\section{Literature Cited}

Evans, R.A., and R.M. Love. 1957. The step-point method of sampling-a practical tool in range research. J. Range Manage. 10: 208-212.

Johnson, C.W., and C.L. Hanson. 1976. Sediment sources and yields from sagebrush rangeland watersheds. In: Proc. Third Interagency Sedimentation Conf., Denver, Colo. p 1-71 to 1-80.

Jurinak, J.J., W.J. Grenney, G.L. Wooldridge, J.P. Riley, and R.J. Wagenet. 1977. A model of environmental transport of heavy metals originating from stack derived particulate emissions in semiarid regions. Report prepared for Southern California Edison Company, Contract
Table 5. Computed yearly soil loss (metric tonnes/ha/yr) at ungrazed big sagebrush sites with sagebrush eradication treatments.

\begin{tabular}{|c|c|c|c|c|c|}
\hline \multirow[b]{2}{*}{ Site and treatment } & \multicolumn{4}{|c|}{ Year } & \multirow{2}{*}{$\begin{array}{l}\text { 4-Year } \\
\text { average }\end{array}$} \\
\hline & 1972 & 1973 & 1974 & 1975 & \\
\hline $\begin{array}{l}\text { Nancy } \\
\text { Sprayed } \\
\text { Cut }\end{array}$ & $\begin{array}{l}0.52 \\
0.52 \\
0.92\end{array}$ & $\begin{array}{c}0.63 \\
0.76 \\
1.10\end{array}$ & $\begin{array}{c}0.49 \\
0.63 \\
0.90\end{array}$ & $\begin{array}{c}0.58 \\
0.60 \\
0.94\end{array}$ & $\begin{array}{c}0.56 \\
0.63 \mathrm{NS} \\
0.97^{* *}\end{array}$ \\
\hline $\begin{array}{l}\text { Whiskey Hill } \\
\text { Untreated } \\
\text { Sprayed }\end{array}$ & $\begin{array}{l}0.76 \\
0.87\end{array}$ & $\begin{array}{l}0.74 \\
1.61\end{array}$ & $\begin{array}{l}0.69 \\
0.74\end{array}$ & $\begin{array}{l}0.63 \\
0.54\end{array}$ & $\begin{array}{c}0.71 \\
0.94 \mathrm{NS}\end{array}$ \\
\hline $\begin{array}{l}\text { Upper Sheep, N.F. } \\
\text { Untreated } \\
\text { Sprayed } \\
\text { Cut }\end{array}$ & $\begin{array}{l}0.65 \\
1.79 \\
3.92\end{array}$ & $\begin{array}{l}1.64 \\
2.44 \\
4.57\end{array}$ & $\begin{array}{l}1.14 \\
1.30 \\
1.95\end{array}$ & $\begin{array}{l}.081 \\
1.48 \\
3.43\end{array}$ & $\begin{array}{c}1.06 \\
1.75 \mathrm{NS} \\
3.47^{* *}\end{array}$ \\
\hline $\begin{array}{l}\text { Reynolds Mtn., E. } \\
\text { Untreated } \\
\text { Sprayed } \\
\text { Cut }\end{array}$ & $\begin{array}{l}0.69 \\
0.78 \\
0.85\end{array}$ & $\begin{array}{l}0.74 \\
1.14 \\
0.94\end{array}$ & $\begin{array}{l}1.28 \\
1.23 \\
1.14\end{array}$ & $\begin{array}{l}0.74 \\
0.99 \\
1.72\end{array}$ & $\begin{array}{l}0.86 \\
1.04 \mathrm{NS} \\
1.16 \mathrm{NS}\end{array}$ \\
\hline $\begin{array}{l}\text { All Sites Combined } \\
\text { Untreated } \\
\text { Sprayed } \\
\text { Cut }\end{array}$ & $\begin{array}{l}0.65 \\
0.99 \\
1.90\end{array}$ & $\begin{array}{l}0.94 \\
1.50 \\
2.20\end{array}$ & $\begin{array}{l}0.90 \\
0.99 \\
1.32\end{array}$ & $\begin{array}{l}0.69 \\
0.90 \\
2.04\end{array}$ & $\begin{array}{l}0.80 \\
1.10 \mathrm{NS} \\
1.87^{* *}\end{array}$ \\
\hline
\end{tabular}

NS Difference between untreated and sprayed treatment means not significant $(P>.05)$.

** Difference between untreated and cut treatment means significant $(P<.01)$.

Table 6. Measured yearly watershed sediment yield (metric tonnes/ha/yr) and computed soil loss by the USLE, grazed areas.

\begin{tabular}{lcccccc}
\hline \hline & \multicolumn{3}{c}{ Watershed } & & Study sites & \\
\cline { 2 - 4 } & $\begin{array}{c}\text { Area } \\
\text { (ha) }\end{array}$ & $\begin{array}{c}\text { Measured } \\
\text { sediment }\end{array}$ & $\begin{array}{c}\text { USLE } \\
\text { yield }\end{array}$ & Soil loss & $\begin{array}{c}\text { USLE } \\
\text { soil loss }\end{array}$ & $\begin{array}{c}\text { Sediment } \\
\text { delivery } \\
\text { ratio }\end{array}$ \\
\hline Whiskey & 48.2 & 0.29 & 1.84 & 0.75 & 0.16 \\
Lower Sheep & 13.4 & 0.22 & 0.47 & 0.89 & 0.47 \\
Upper Sheep & 25.7 & 0.31 & 2.11 & $3.24^{3}$ & 0.15 \\
Reynolds Mtn. & 40.4 & 0.43 & 1.93 & $0.86^{3}$ & 0.22 \\
\hline
\end{tabular}

'Soil loss was computed using USLE factors representing the total watershed.

${ }^{2}$ Soil loss was computed using USLE factors from data at study sites within the watershed.

${ }^{3}$ Average of two grazed sites.

No. U0966901, Utah State University, Logan, Utah. 143 p.

McCool, D.K., R.I. Papendick, and F.L. Brooks. 1976. The universal soil loss equation as adapted to the Pacific Northwest. In: Proc. Third Interagency Sedimentation Conf., Denver, Colo. p 2-135 to 2-147.

Miller, J.F., R.H. Frederick, and R.J. Tracey. 1973. Precipitationfrequency atlas of the western United States. Volume V-Idaho. U.S. Dep. Commerce. NOAA Atlas 2, 43 p.

Schumaker, G.A., and C.L. Hanson. 1977. Herbage response after mechanical and herbicide treatment of big sagebrush in southwest Idaho. U.S. Dep. Agriculture, Western Region, ARS W-46, 15 p.

Stephenson, G.R. 1977. Soil-geology-vegetation inventories for Reynolds Creek Watershed. Idaho Agr. Exp. Sta. Misc. Ser. No. 42, 73 p.

Tisdale, E.W., M. Hironaka, and M.A. Fosberg. 1969. The sagebrush region in Idaho. Idaho Agr. Exp. Sta. Bull. 152. 15 p.

U.S. Department of Agriculture. 1974. Soil erodibility and soil loss tolerance factors for Idaho soils. Soil Conserv. Services Tech. Note-Agron., No. 19, Boise, Ida. 35 p.

U.S. Department of Agriculture. 1975. Procedure for computing sheet and rill erosion on project areas. Tech. Release No. 51 (Rev.) Geol. 16 p.

Wischmeier, W.H. 1975. Estimating the soil loss equation's cover and management factor for undisturbed areas. In: Proc. Sediment-Yield Workshop, Oxford, Miss. Nov. 1972. U.S. Dep. Agr., ARS-S-40, p. 118-124.

Wischmeier, W.H. 1976. Use and misuse of the universal soil loss equation. J. Soil and Water Conserv. 31(1): 5-9.

Wischmeier, W.H., and D.D. Smith. 1978. Predicting rainfall erosion losses-A guide to conservation planning. U.S. Dep. Agr., Agr. Handbk No. $537.58 \mathrm{p}$ 